\title{
A variational approach to retrieve rain rate by combining information from rain gauges, radars, and microwave links
}

Article

Published Version

Bianchi, B., Van Leeuwen, P., Hogan, R. and Berne, A. (2013) $A$ variational approach to retrieve rain rate by combining information from rain gauges, radars, and microwave links. Journal of Hydrometeorology, 14 (6). pp. 1897-1909. ISSN 1525-7541 doi: https://doi.org/10.1175/JHM-D-12-094.1 Available at https://centaur.reading.ac.uk/40384/

It is advisable to refer to the publisher's version if you intend to cite from the work. See Guidance on citing.

Published version at: http://dx.doi.org/10.1175/JHM-D-12-094.1

To link to this article DOI: http://dx.doi.org/10.1175/JHM-D-12-094.1

Publisher: American Meteorological Society

All outputs in CentAUR are protected by Intellectual Property Rights law, including copyright law. Copyright and IPR is retained by the creators or other copyright holders. Terms and conditions for use of this material are defined in the End User Agreement.

www.reading.ac.uk/centaur 
Central Archive at the University of Reading

Reading's research outputs online 


\title{
A Variational Approach to Retrieve Rain Rate by Combining Information from Rain Gauges, Radars, and Microwave Links
}

\author{
BLANDINE BIANCHI \\ Environmental Remote Sensing Laboratory, Civil and Environmental Engineering, School of Architecture, \\ École Polytechnique Fédérale de Lausanne, Lausanne, Switzerland \\ PETER JAN VAN LEeUwen AND Robin J. Hogan \\ Department of Meteorology, University of Reading, Reading, United Kingdom
}

\section{AleXIs BERNE}

Environmental Remote Sensing Laboratory, Civil and Environmental Engineering, School of Architecture, École Polytechnique Fédérale de Lausanne, Lausanne, Switzerland

(Manuscript received 26 June 2012, in final form 3 May 2013)

\begin{abstract}
Accurate and reliable rain rate estimates are important for various hydrometeorological applications. Consequently, rain sensors of different types have been deployed in many regions. In this work, measurements from different instruments, namely, rain gauge, weather radar, and microwave link, are combined for the first time to estimate with greater accuracy the spatial distribution and intensity of rainfall. The objective is to retrieve the rain rate that is consistent with all these measurements while incorporating the uncertainty associated with the different sources of information. Assuming the problem is not strongly nonlinear, a variational approach is implemented and the Gauss-Newton method is used to minimize the cost function containing proper error estimates from all sensors. Furthermore, the method can be flexibly adapted to additional data sources. The proposed approach is tested using data from 14 rain gauges and 14 operational microwave links located in the Zürich area (Switzerland) to correct the prior rain rate provided by the operational radar rain product from the Swiss meteorological service (MeteoSwiss). A cross-validation approach demonstrates the improvement of rain rate estimates when assimilating rain gauge and microwave link information.
\end{abstract}

\section{Introduction}

The problem of accurate measurement of rainfall intensity has been long investigated because it has important implications in meteorology, agriculture, environmental policies, monitoring of sewage systems in urban areas, and weather forecasting. Over past decades, various techniques have been developed for monitoring rainfall, but its strong spatial and temporal variability still represents a significant source of uncertainty. In this study, a variational approach is proposed

Corresponding author address: Alexis Berne, Environmental Remote Sensing Laboratory, Civil and Environmental Engineering, School of Architecture, Ecole Polytechnique Fédérale de Lausanne, GR C2 564, Station 2, CH-1015 Lausanne, Switzerland.

E-mail: alexis.berne@epfl.ch to retrieve the rain rate combining measurements from rain gauges, weather radars, and microwave links in order to obtain reliable and accurate rain rate estimates.

\section{a. Rain gauges}

Rain gauges have a relatively high accuracy but collect information at the point scale. Because of the spatial variability of rainfall, they have limited spatial representativity, in particular at short time scales. Several potential measurement error sources can affect the accuracy of rain gauge data (e.g., Nespor and Sevruk 1999; Upton and Rahimi 2003; Sieck et al. 2007). Their effect can, however, be reduced by carefully choosing the rain gauge location, far from the obstacles and from the sources of heat (e.g., World Meteorological Organization 2008). Despite these limitations, rain gauges are still able to provide useful estimates of rainfall intensity, and 
because of the direct measurement principle, their records are often considered as ground truth.

\section{b. Weather radar}

Rainfall intensity can be estimated from the radar reflectivity with the following relationship:

$$
Z=a r^{b},
$$

where $r$ is the rainfall intensity $\left(\mathrm{mm} \mathrm{h}^{-1}\right)$ and $Z$ is the radar reflectivity $\left(\mathrm{mm}^{6} \mathrm{~m}^{-3}\right)$. Different parameterizations of $a$ and $b$ have been proposed in the literature (e.g., Battan 1973). This relationship is, however, merely empirical and is subject to uncertainties that are mostly due to the rainfall microstructure. To increase the accuracy, dual-polarization weather radar systems have been developed (Bringi and Chandrasekar 2001). Taking advantage of the nonspherical shape of large rain drops, such systems allow for the improvement of hydrometeor identification and rain rate estimation (Meischner et al. 1991; Ryzhkov et al. 2005; Hogan 2007).

During its propagation, the radar signal can be attenuated by heavy rain (at $\mathrm{X}$ and $\mathrm{C}$ band) or contaminated by nonmeteorological echoes. Various source of uncertainty can affect radar rain rate estimates (attenuation at $\mathrm{C}$ and $\mathrm{X}$ band, light band contamination, and ground clutter, to list a few). Operational services have developed and operationally implemented quality control and correction procedures to minimize these uncertainties (Germann et al. 2006).

Finally, it is emphasized that radar gives a measure of observable quantities on the whole spatial area and reveals the complete structure of the meteorological phenomenon. The integration with other sensors, such as those specific to the ground, is a way to improve rain rate estimation. Often, this is done using rain gauges (e.g., Goudenhoofdt and Delobbe 2008). Radar data (radial velocity and reflectivity) also start to be assimilated in operational NW models (Caumont et al. 2010).

\section{c. Microwave links}

An alternative approach for rain rate measurement has been recently proposed using the attenuation because of rain affecting the microwave signals employed for wireless data exchange (Messer et al. 2006; Leijnse et al. 2007).

The wide use of microwave communication technology makes the already-installed facilities attractive for precipitation inferences. The links send and receive information that is carried on microwave signals, with frequencies between 10 and $60 \mathrm{GHz}$. At these frequencies, the wavelength is comparable to the drop sizes and causes an attenuation of the received signal power due to the scattering and absorption by the raindrops. Because the total attenuation along the link can be measured, it is possible to get an estimate of the path-averaged rain rate through the following power law equation (Atlas and Ulbrich 1977; Olsen et al. 1978):

$$
k=\alpha r^{\beta},
$$

where $k\left(\mathrm{~dB} \mathrm{~km}^{-1}\right)$ is the specific attenuation of the microwave signal; $r$ is the rainfall intensity; and the parameters $\alpha$ and $\beta$ depend on the frequency, polarization, drop size distribution (DSD), and temperature. When the frequency is about $35 \mathrm{GHz}, \beta$ is close to 1 and Eq. (2) becomes linear (Atlas and Ulbrich 1977; Berne and Uijlenhoet 2007). Similar to radar, microwave links provide indirect measurements of rain rate. They also integrate rain rates over a few kilometers (typical path length). Their advantage is the fact that $\beta$ of Eq. (2) is closer to 1 than $b$ of Eq. (1), which is around 1.6. Hence, microwave links nicely complement rain gauge and radar networks.

Several studies have been conducted on microwave links specifically designed for rainfall monitoring: D'Amico et al. (2003) explored the use of fixed dualfrequency microwave links, to overcome the problem if $\beta \neq 1$, to estimate the path-averaged rain rate along a link. Rahimi et al. $(2003,2004)$ presented some results showing the potential of dual-frequency microwave links for measuring rainfall intensity in urban locations. These results were found highly concordant with the rainfall estimates obtained from local rain gauges and weather radars. Krämer and Verwon (2005) proposed a correction of X-band radar rainfall estimates using a link. Along the link path, some rain gauges were used to evaluate the path-averaged rain rate estimates, showing reasonably good agreement with the corrected radar measurements.

The estimation of the spatial distribution of rainfall intensity distribution from the received signal level recorded by commercial microwave links has been proposed by Zinevich et al. (2008) and Goldshtein et al. (2009). The technique proposed by Zinevich et al. (2008) is better suited for dense networks, where the assumption of constant rainfall over a pixel is valid, since it explicitly accounts for variations of rainfall intensity between different pixels along the link paths. However, it is a deterministic algorithm that does not assume any observation uncertainty. The approach proposed by Goldshtein et al. (2009) seems more appropriate for sparse networks, because the assumption of constant rainfall over a link segment is weaker than that in Zinevich et al. (2008) and it explicitly accounts for the observation uncertainty. Other interesting results were obtained by 
Zinevich et al. (2009): their method describes the evolution of the rain rate field in time, assuming that during short time intervals the main force acting on a rain cell is advection. The estimation of the spatial distribution of rain rate along the direction of motion was done by applying an extended Kalman filter over the system of links to generate instantaneous reconstruction of the rain field. This dynamic approach can be adapted to incorporate data from other sources such as rain gauges and radars as proposed by Grum et al. (2005).

\section{d. Objective of this paper}

The main objective of this work is to take advantage of the three main sources of information about rainfall intensity (i.e., rain gauges, weather radars, and telecommunication microwave links) by combining the different measurements through a static optimal retrieval technique in order to obtain reliable and accurate rain rate estimates over a given area, together with the associated uncertainties. Each source of information has its drawbacks, but combining them will lead to an improved rain rate estimation.

In section 2, the static optimal retrieval with a variational approach for combining different sources of information to retrieve the rain rate is described. In section 3, the study area in Zürich, Switzerland, is presented. The retrieved rain rates obtained for three events are then evaluated. The conclusions are given in section 4 .

\section{Optimal retrieval with a variational approach}

\section{a. Optimal estimation theory}

Data assimilation is widely used in many environmental fields, in particular in weather modeling and forecasting (e.g., Chahine et al. 2006) and in hydrological modeling (e.g., McLaughlin 2002), but the latest developments have not been explored for merging rainfall information gathered by rain gauges, microwave links, and radars. In static (i.e., time independent) data assimilation, we need a priori knowledge of the state we want to estimate (e.g., the $2 \mathrm{D}$ distribution of rainfall intensity) and a forward model, used to compare the forward observations (obtained using the state estimate) with the true observations.

Then, assuming that the process is static and the relationship between the observations and the state is represented by a nonlinear forward model $h$, the system is defined as follows:

$$
\begin{aligned}
& \mathbf{x}=\mathbf{x}^{b}+\boldsymbol{\epsilon}, \\
& \mathbf{y}=h(\mathbf{x})+\boldsymbol{\eta},
\end{aligned}
$$

where $\mathbf{x}$ is the state vector of dimension $N_{x}$, that is, the vector of variables we want to estimate, and $\mathbf{y}$ is the observation vector of dimension $N_{y}$, that is, the vector of measurements. We denote with $\mathbf{x}^{b}$ the prior or background state, $\boldsymbol{\epsilon}$ is the instantaneous background error, and $\boldsymbol{\eta}$ is the instantaneous observation error.

The presence of error in both the forward model and the observations leads to a probabilistic formulation of the problem. The probability of a state $\mathbf{x}$ can be represented by a probability density function $p(\mathbf{x})$. We are looking for an updated estimate that takes into account the information provided by the prior and the likelihood [probability distribution for the observation conditional on the state $p(\mathbf{y} \mid \mathbf{x})]$. Bayes' rule generates this update as

$$
p(\mathbf{x} \mid \mathbf{y})=\frac{p(\mathbf{y} \mid \mathbf{x}) p(\mathbf{x})}{p(\mathbf{y})}
$$

where $p(\mathbf{x} \mid \mathbf{y})$ is the posterior probability of the state $\mathbf{x}$ given $\mathbf{y}, p(\mathbf{y} \mid \mathbf{x})$ is the likelihood, and $p(\mathbf{x})$ is the prior probability of the state $\mathbf{x}$ (e.g., Rogers 2008).

We want to find the state vector $\mathbf{x}$ such that Eq. (4) is maximized. There are several possible optimal estimates of the state, given the observations. We will assume the errors are normally distributed: $\boldsymbol{\epsilon} \sim \mathcal{N}(0, \mathbf{B})$ and $\boldsymbol{\eta} \sim$ $\mathcal{N}(0, \mathbf{R})$, where $\mathbf{B}$ and $\mathbf{R}$ are the covariance matrix of the prior state and of the observation error, respectively. Then, finding the state $\mathbf{x}$ such that $p(\mathbf{x} \mid \mathbf{y})$ is maximum is equal to finding the state $\mathbf{x}$ such that the cost function

$$
J(\mathbf{x})=\left(\mathbf{x}-\mathbf{x}^{b}\right)^{\mathrm{T}} \mathbf{B}^{-1}\left(\mathbf{x}-\mathbf{x}^{b}\right)+[\mathbf{y}-h(\mathbf{x})]^{\mathrm{T}} \mathbf{R}^{-1}[\mathbf{y}-h(\mathbf{x})]
$$

is minimized, where the matrices $\mathbf{B}^{-1}$ and $\mathbf{R}^{-1}$ assign more or less weight to the prior state estimate and observations, depending on how confident we are in the quality of the observations and the prior state estimate. The cost function defined in Eq. (5) cannot be minimized in one step because of the nonlinear forward model $h(\mathbf{x})$; we apply the Gauss-Newton method in which a linearized version of the cost function is minimized iteratively. At iteration $k$ we have an estimate of the state vector $\mathbf{x}_{k}$ and the corresponding forward observations $h\left(\mathbf{x}_{k}\right)$. The linearized cost function is obtained replacing $h(\mathbf{x})$ by

$$
h(\mathbf{x}) \simeq h\left(\mathbf{x}_{k}\right)+\hat{\mathbf{H}}\left(\mathbf{x}-\mathbf{x}_{k}\right),
$$

where $\hat{\mathbf{H}}=\partial h(\mathbf{x}) / \partial \mathbf{x}$ is the Jacobian, a matrix containing the partial derivative of each observation with respect to each element of the state vector, due to the linearization of the operator $h$. The Jacobian is calculated at each iteration of the optimization problem. Indeed, 
minimizing Eq. (5) is equivalent to solving the optimization problem

$$
\mathbf{x}_{k+1}=\mathbf{x}_{k}+\mathbf{A}^{-1}\left[\hat{\mathbf{H}}^{\mathrm{T}} \mathbf{R}^{-1} \delta \mathbf{y}-\mathbf{B}^{-1}\left(\mathbf{x}_{k}-\mathbf{x}^{b}\right)\right],
$$

where $\delta \mathbf{y}=\mathbf{y}-h\left(\mathbf{x}_{k}\right)$ and $\mathbf{A}$ denotes the Hessian, which can be calculated as follows:

$$
\mathbf{A}=\mathbf{B}^{-1}+\hat{\mathbf{H}}^{\mathrm{T}} \mathbf{R}^{-1} \hat{\mathbf{H}} .
$$

A more detailed description of the variational approach can be found in Rogers (2008).

\section{b. Formulation of the variational problem}

In this section, the two-dimensional variational problem is described. The state vector we want to retrieve is given by

$$
\mathbf{x}=\left[\begin{array}{c}
\ln (\mathbf{r}) \\
\ln (\boldsymbol{\alpha})
\end{array}\right]
$$

which is the logarithm of the rain rate $r_{i}$ at the $i$ th pixel and the value $\alpha_{q_{i}}$ of the $k-r$ relationship [see Eq. (2)] of the $q$ th microwave link at the $i$ th pixel covered by the link. By using the logarithm, the possibility of retrieving unphysical negative values is avoided. The distribution of rain rate is highly skewed, and it is common practice to assume it to be lognormal (Sauvageot 1994). The zeros in the prior are set to $10^{-2}\left(\mathrm{~mm} \mathrm{~h}^{-1}\right)$, which is at least 10 times smaller than the typical minimum value recorded by the radar and the rain gauges. We choose to retrieve also the $\log$ of the prefactor $\alpha$ of the $k-r$ power law above each pixel for each link such that the variation along the path is properly represented and, in this way, the path integrating characteristics of the rain rate information provided by the links are properly represented. The prefactor $\alpha$ can be related to the uncertainty in the DSD, but in practice it can account for many other errors (e.g., wet antenna attenuation, atmospheric attenuation, and temperature effect on the electronics).

The background state estimate $\mathbf{x}^{b}$ is the rain rate product obtained from the radar network (provided by MeteoSwiss). The parameter $\alpha$ in Eq. (2) is estimated using $1 \mathrm{yr}$ of disdrometer data collected in Lausanne, Switzerland (Jaffrain et al. 2011), to simulate the attenuation values measured by the microwave links as a function of the drop size using the T-matrix approach, similar to Schneebeli and Berne (2012). We decided to use the rain rate estimated by the operational radars as prior for three main reasons: (a) radar is largely employed for weather forecasts and hence easily and commonly accessible, (b) the convergence is faster than with a climatological prior since the data are available at every grid point over the whole area, and (c) operational weather services (MeteoSwiss in our case) have put a lot of effort in cleaning radar data and correcting for the main sources of errors (e.g., Germann et al. 2006).

Since the state vector in Eq. (9) is in terms of the natural logarithm of the rain rate, the normal assumption for the errors in $\mathbf{x}$ is equivalent to the lognormal assumption for the distribution of errors in rain rate [for an alternative approach see for example Fletcher and Zupanski (2006)].

To obtain the forward rain gauge and microwave link observations, after exponentiating the state values, we use as the forward model for the gauge located in the pixel $i$ :

$$
r_{g_{i}}=r_{i}
$$

and for the link located above the pixel $i$ :

$$
k_{i}=\alpha_{i} r_{i}^{\beta},
$$

and to obtain the total attenuation:

$$
K=\sum_{i=1}^{l} u_{i} k_{i}
$$

where $u_{i}$ is the length of the link in the $i$ th pixel, so $\sum_{i} u_{i}=L$, with $L$ as the length of the link.

The observation vector is

$$
\mathbf{y}=\left[\begin{array}{c}
\mathbf{r}_{g} \\
\ln (\mathbf{K})
\end{array}\right]
$$

where $\mathbf{r}_{g}$ is the vector of rain rate recorded by $m$ rain gauges $\left(\mathrm{mm} \mathrm{h}^{-1}\right)$ and $\mathbf{K}$ is the vector of total attenuation recorded by the $n$ microwave links. We choose to use the rain gauge observations in linear rain rate to incorporate the zero in the algorithm.

The cost function defined in Eq. (5) involves the error covariance matrices of the observations $\mathbf{R}$ and of the background $\mathbf{B}$, which must be known a priori and play an important role in the variational problem; their relative values control the relative influences of the observations and of the background. For instance, high confidence in the background will be expressed by choosing B smaller than $\mathbf{R}$, thus putting a heavier penalty on deviations from the background than on deviations from the observations. Similarly, if the matrix $\mathbf{R}$ has very small elements, we would retrieve the observations exactly. 


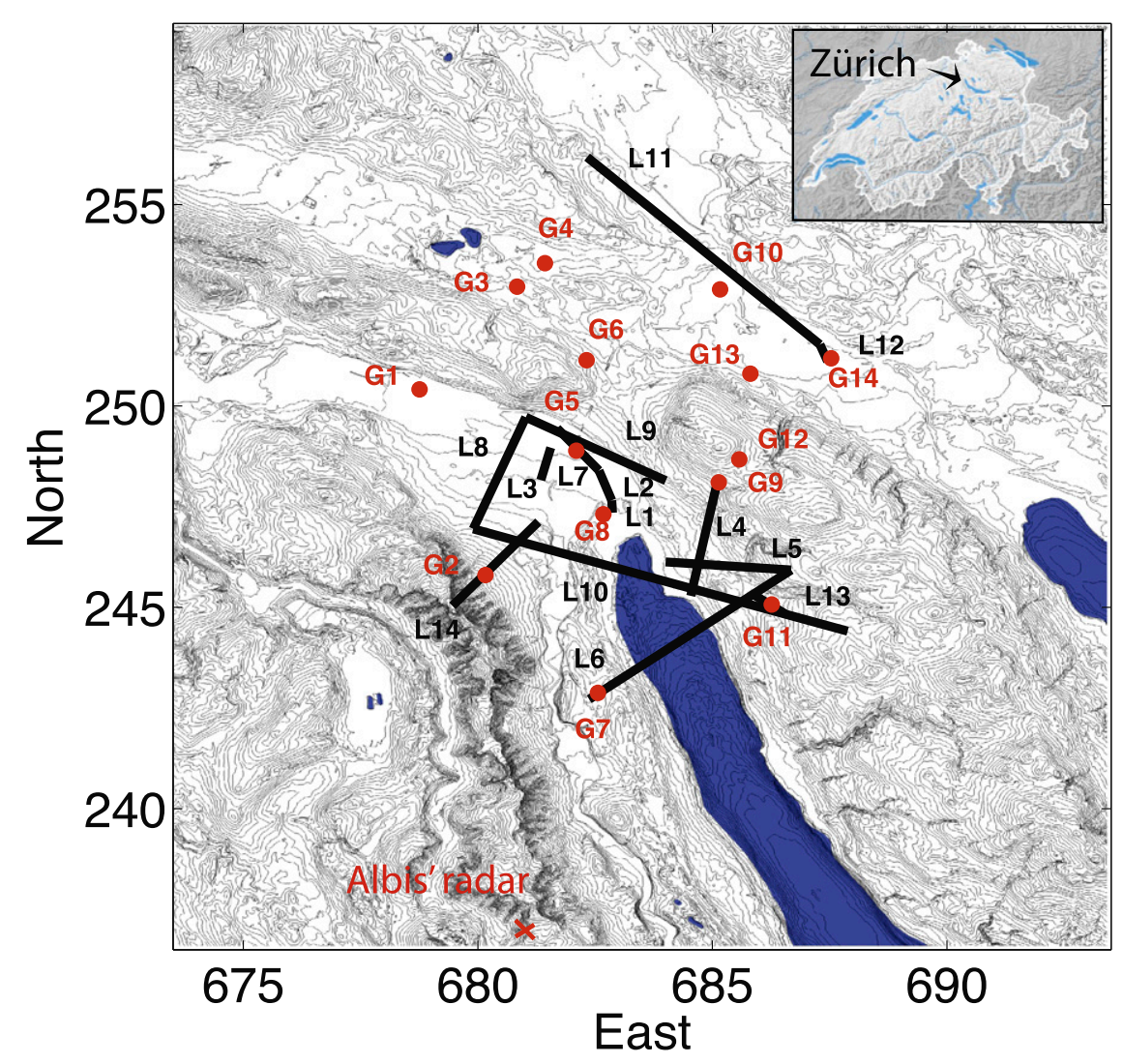

FIG. 1. Locations of the microwave links, rain gauges, and Albis's radar (in Swiss grid) in the area of Zürich, Switzerland.

\section{Application}

The study area and the data are first described. The parameterization of the error covariance matrices $\mathbf{B}$ and $\mathbf{R}$ is then detailed. Then proposed method is finally applied for three rain events, and the quality of the retrieved rain rate is evaluated by a cross validation.

\section{a. Study area and data}

The variational technique is applied on a set of data collected in the Zürich area (Switzerland). The select area of $20 \times 23 \mathrm{~km}^{2}$ is mainly covered by the Albis radar managed by MeteoSwiss, which is located at a distance of about $15 \mathrm{~km}$ from Zürich. This is a relatively flat area with few hills, the highest peak is close to the radar location at $928 \mathrm{~m}$ above sea level. The rain rate recorded by 14 rain gauges, and the total attenuation of 14 operational telecommunication microwave links managed by the company Orange, are used to correct the prior rain rate provided by the operational radar network managed by MeteoSwiss over the same area (at a resolution of $1 \times 1 \mathrm{~km}^{2}$ every $5 \mathrm{~min}$ ), as illustrated in Fig. 1 .

The rain gauge network is composed of 13 tippingbucket gauges [from Entsorgung+Recycling Zürich
(ERZ) and MeteoSwiss] and one weighing gauge [from the Swiss Federal Institute of Aquatic Science and Technology (EAWAG)] at different time resolution. To make the data from rain gauge, microwave link, and radar comparable, rain gauge measurements have been resampled at $5 \mathrm{~min}$. This resampling assumes a uniform distribution within the original time step. The value for the new time step is obtained by summing the respective proportion in the covered original time steps.

The microwave links used in this application work at 23,38 , and $58 \mathrm{GHz}$ at horizontal or vertical polarization and with a power resolution of 0.1 or $1 \mathrm{~dB}$. The microwave links have been resampled similarly to the rain gauge and preprocessed to remove erroneous records as well. The microwave link characteristics are summarized in Table 1. To obtain the attenuation from the received signal level (RSL) assuming the transmitted power is constant, we adopt the simple approach proposed by Leijnse et al. (2007): the attenuation baseline, that is, the attenuation when there is no rain, is defined as the mode of the measured RSL over a sufficiently long period (typically a few months). Moreover, the wet antenna contribution should be considered to avoid biases in the rain rate values derived from microwave 
TABLE 1. Frequency $(\mathrm{GHz})$, polarization [horizontal $(\mathrm{H})$ or vertical $(\mathrm{V})]$, length $(\mathrm{km})$, and power resolution $(\mathrm{dB})$ of the 14 microwave links.

\begin{tabular}{|c|c|c|c|c|c|c|c|c|c|c|c|c|c|c|}
\hline Link & 1 & 2 & 3 & 4 & 5 & 6 & 7 & 8 & 9 & 10 & 11 & 12 & 13 & 14 \\
\hline Frequency & 58 & 38 & 38 & 38 & 38 & 23 & 38 & 23 & 23 & 23 & 23 & 58 & 38 & 38 \\
\hline Polarization & $\mathrm{V}$ & $\mathrm{H}$ & $\mathrm{H}$ & $\mathrm{V}$ & $\mathrm{H}$ & $\mathrm{H}$ & $\mathrm{H}$ & $\mathrm{H}$ & V & $\mathrm{V}$ & $\mathrm{H}$ & $\mathrm{V}$ & $\mathrm{H}$ & $\mathrm{H}$ \\
\hline Length & 0.3 & 0.8 & 0.8 & 2.9 & 2.7 & 5.4 & 1.4 & 3.0 & 3.4 & 8.4 & 6.8 & 0.5 & 2.8 & 0.8 \\
\hline Power resolution & 0.1 & 1 & 0.1 & 0.1 & 0.1 & 0.1 & 0.1 & 1 & 1 & 0.1 & 0.1 & 0.1 & 1 & 1 \\
\hline
\end{tabular}

link; following Overeem et al. (2011), the average of the wet antenna attenuation is set to $1.5 \mathrm{~dB}$.

\section{b. Parameterization of the covariance matrices}

To compute the error covariance matrices $\mathbf{R}$ and $\mathbf{B}$, in our application of size $(28 \times 28)$ and $(526 \times 526)$ representatively (we retrieve 460 rain rate values above each pixel plus $66 \alpha$ values), we use a period of 5 months, from June to October 2009, for which data from the three types of instruments are available.

We assume that the covariance matrix of the observation error $\mathbf{R}$ is a diagonal matrix of dimension $N_{y} \times$ $N_{y}$, with $N_{y}=m+n$, given by the errors in the rain gauge and microwave link observations, so that they are not spatially correlated. A similar assumption for radar data has been made by Hogan (2007) and is common in data assimilation applications. The elements $R_{i, i}$ correspond to the square of the standard error in rain gauge and microwave link observations.

The first $m$ elements of the diagonal correspond to the rain gauge measurement error $R_{i i} \equiv\left[\Delta\left(r_{g_{i}}\right)\right]^{2}=\sigma_{g_{i}}^{2}$. Taylor series for the logarithm leads to $\Delta r_{g_{i}} \approx \Delta \ln r_{g} r_{g_{i}}$ in linear rain rate, and $\Delta \ln r_{g}$ is estimated as follows:

$$
\overline{\left(\ln r_{g_{1}}-\ln r_{g_{2}}\right)^{2}}=\overline{\left[\ln r_{g_{1}}-A-\left(\ln r_{g_{2}}-A\right)\right]^{2}},
$$

where $\ln r_{g_{1}}$ and $\ln r_{g_{2}}$ are the natural logarithm of the rain rate collected by two rain gauges less than $1 \mathrm{~km}$ apart during $1 \mathrm{yr}$ and $A$ is the average rain rate (in $\log$ ) on an area of $1 \mathrm{~km}^{2}$. Since rain gauge errors are assumed to be uncorrelated, $\overline{\left(\ln r_{g_{1}}-A\right)\left(\ln r_{g_{2}}-A\right)}$ is zero and

$$
\overline{\left(\ln r_{g_{1}}-\ln r_{g_{2}}\right)^{2}}=\overline{\left(\ln r_{g_{1}}-A\right)^{2}+\left(\ln r_{g_{2}}-A\right)^{2}} .
$$

If we assume the rain gauge error is the same for the two rain gauges, then

$$
\Delta \ln r_{g}=\sqrt{\frac{\overline{\left(\ln r_{g_{1}}-\ln r_{g_{2}}\right)^{2}}}{2}} .
$$

In our case we obtain $\Delta \ln r_{g}=0.58$, so $\Delta r_{g_{i}} \approx 0.58 r_{g_{i}}$ in linear rain rate.

If the rain rate is less than $1.7 \mathrm{~mm} \mathrm{~h}^{-1}$, then the rain gauge error is quantified as the standard deviation of a uniform distribution $\Delta / \sqrt{12}=0.34 \mathrm{~mm} \mathrm{~h}^{-1}$, where $\Delta=$ $1.2 \mathrm{~mm} \mathrm{~h}^{-1}$ is the quantization step of the rain rate value recorded by a tipping-bucket rain gauge at a time resolution of $5 \mathrm{~min}$. It is emphasized that this includes the representativeness error when considering point values as representative of a $1 \times 1 \mathrm{~km}^{2}$ area.

The other $n$ diagonal elements correspond to the total attenuation measurement error $R_{i i} \equiv\left[\Delta \ln \left(K_{q}\right)\right]^{2}=\sigma_{K_{q}}^{2}$, given by:

$$
\begin{aligned}
& \Delta \ln \left(K_{q}\right) \simeq 0.8 K_{q}^{-1} \quad \text { for the link at } 0.1 \mathrm{~dB}, \\
& \Delta \ln \left(K_{q}\right) \simeq 1.2 K_{q}^{-1} \quad \text { for the link at } 1 \mathrm{~dB},
\end{aligned}
$$

where $K_{q}$ is the total attenuation of link $q$. The total attenuation errors $\Delta K_{q}=0.8$ and $1.2 \mathrm{~dB}$ include the atmospheric gases attenuation, the quantization error, and other contamination sources of errors (e.g., temperature effect on the electronics). These values have been derived in Bianchi et al. (2013).

The covariance matrix of the background error also needs to be parameterized:

$$
\mathbf{B}=\left(\begin{array}{ccccccc}
B_{11} & B_{12} & \cdots & 0 & 0 & \cdots & 0 \\
B_{21} & B_{22} & \ddots & \vdots & \vdots & \ddots & \vdots \\
\vdots & \ddots & \ddots & B_{(s-1) s} & \vdots & \ddots & \vdots \\
0 & \cdots & B_{s(s-1)} & B_{s s} & 0 & \cdots & 0 \\
0 & \cdots & \cdots & 0 & B_{(s+1)(s+1)} & \cdots & 0 \\
\vdots & \ddots & \ddots & \vdots & \vdots & \ddots & \vdots \\
0 & \cdots & \cdots & 0 & 0 & \cdots & B_{(s+p)(s+p)}
\end{array}\right) .
$$


Here, $s$ is the total number of pixels where the rain rates are retrieved and $p$ is the total number of pixels covered by the microwave links, hence, $N_{x}=s+p$. The path of each link has been divided into segments defined by the length of its path over each pixel. The path-averaged rain rate is computed as the sum of the different pixel values weighted by the respective length of each segment.

The first quadrant represents the error of the radar in estimating the rain rate since this information is used as prior. To estimate these first elements, we assume $B_{i i} \equiv\left[\Delta \ln \left(r_{r_{i}}\right)\right]^{2}=\sigma_{r_{i}}^{2}$, with $i=1, \ldots, s$. For the estimation of the rain rate error that stems from the radar, we adopt the same approach as for $\Delta \ln \left(r_{g}\right)$ by assuming

$$
\overline{\left(\ln r_{g_{*}}-\ln r_{r}\right)^{2}}=\left(\Delta \ln r_{r}\right)^{2}+\left(\Delta \ln r_{g}\right)^{2}
$$

where $\ln r_{g_{*}}$ represents the logarithm of the "true" rain rate values over several months recorded by the rain gauge in the referred radar pixel and $\ln r_{r}$ is the logarithm of the rain rate estimated by the radar: the mean square of the difference of this error series is supposed to be equal to the squared radar error plus the rain gauge representativeness error (assuming they are not correlated). We can compute the radar error with the following expression:

$$
\Delta \ln r_{r}=\sqrt{\overline{\left(\ln r_{g_{1}}-\ln r_{r}\right)^{2}}-\left(\Delta \ln r_{g}\right)^{2}} .
$$

The radar error in Eq. (20) represents the diagonal of the matrix $\mathbf{B}$, but the fact that the radar error is correlated in space also needs to be taken into account (e.g., Berenguer and Zawadzki 2008). Assuming that the rain gauge errors are spatially uncorrelated, we estimate the spatial autocorrelation of the radar error for each couple of rain gauges $\rho_{i j}=\overline{\nu_{i} \nu_{j}}$, where $\nu_{i}$ is given by

$$
\nu_{i}=\frac{\left(\ln r_{g_{i}}-\overline{\ln r_{g_{i}}}\right)-\left(\ln r_{r_{i}}-\overline{\ln r_{r_{i}}}\right)}{\sigma_{\left[\left(\ln r_{g_{i}}-\overline{\ln r_{g_{i}}}\right)-\left(\ln r_{r_{i}}-\overline{\ln r_{r_{i}}}\right)\right]}}
$$

and $r_{r_{i}}$ corresponds to the rain rate estimated by the radar over the pixel $i$. Figure 2 shows the spatial autocorrelation fitted with an inverse exponential model (see section 3a for details on the instruments and their locations).

The first quadrant of the covariance matrix of the state is given by

$$
B_{i j}=B_{i i} \exp \left(-\frac{d_{i j}}{d_{0}}\right),
$$

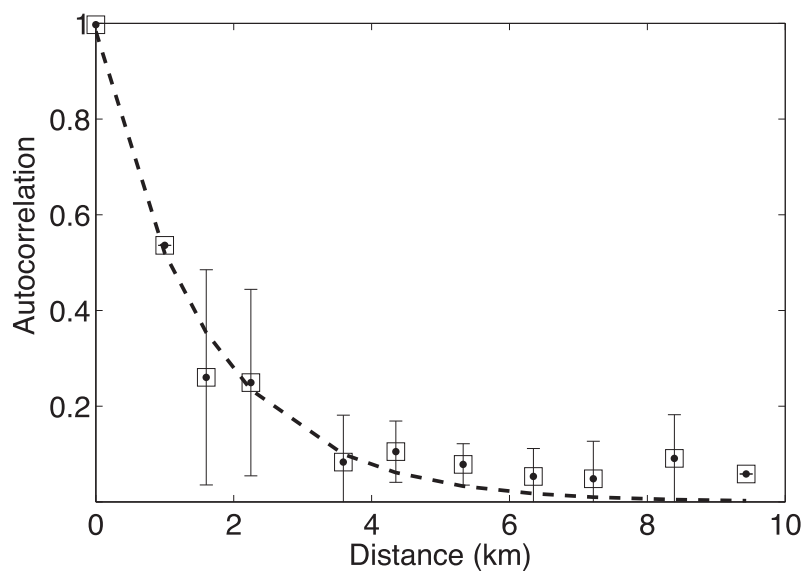

FIG. 2. Correlation of the logarithms of the gauge-radar ratios assuming the rain gauge error is uncorrelated (based on 2 months). The $x$ axis corresponds to the distance between each couple of rain gauge locations. The dashed line indicates the fitted inverse exponential model.

where $B_{i i}=0.68$ is the radar error in estimating the rain rate (in log) obtained from Eq. (20) and $d_{i j}$ is the distance between the pixel $i$ and $j$, with $i, j=1, \ldots, s$; the fitted $e$-folding distance $d_{0}$ in Eq. (22) is about $1.5 \mathrm{~km}$.

From Eq. (2), we have

$$
\ln (\mathbf{k})=\ln (\boldsymbol{\alpha})+\beta \ln (\mathbf{r}) .
$$

To estimate the background of $\boldsymbol{\alpha}$, and to parameterize $\beta$, we used $1 \mathrm{yr}$ of disdrometer data to simulate the attenuation affecting the microwave link as a function of the measured DSD using the T-matrix approach, similar to Schneebeli and Berne (2012). The T-matrix method (Barber and Yeh 1975; Mishchenko et al. 1996) has been used for the calculation of the scattering amplitudes of oblate spheroids where the drop axial ratios were a function of the equivolumetric drop diameter following the models of Andsager et al. (1999), Brandes et al. (2004), and Thurai and Bringi (2005). The refractive index of liquid water was taken from the model of Meissner and Wentz (2004).

The relation between the diameter of the drops $D(\mathrm{~mm})$ provided by the disdrometer and the rain rate $r\left(\mathrm{~mm} \mathrm{~h}^{-1}\right)$ is given by

$$
r=6 \pi 10^{-4} \int_{D_{\min }}^{D_{\max }} D^{3} v(D) N(D) d D,
$$

where $v(D)$ is the fall velocity $\left(\mathrm{m} \mathrm{s}^{-1}\right)$ and $N(D) d D$ is the concentration of drops with diameter between $D$ and $D+d D$. For the terminal fall velocity of the drops, the model of Beard (1977) was adopted. 


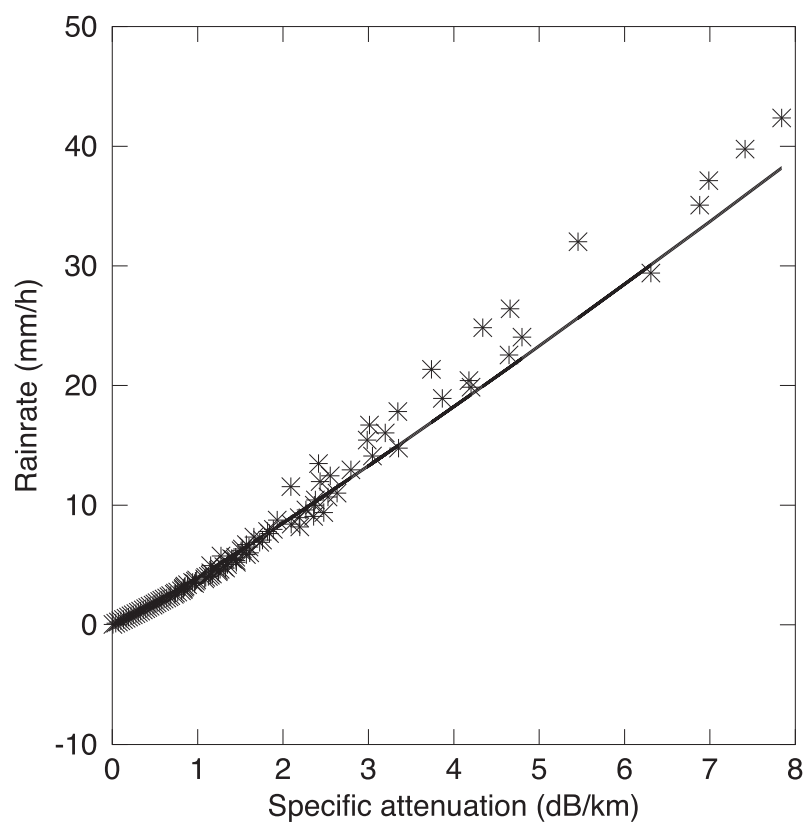

FIG. 3. Simulated specific attenuation at horizontal polarization at a frequency of $38 \mathrm{GHz}$ vs rain rate.

Figure 3 shows the derived rain rate and specific attenuation at a frequency of $38 \mathrm{GHz}$, at horizontal polarization, and at a temperature of $280 \mathrm{~K}$. By fitting these data points, we obtained $\beta=0.93$ and $\alpha=0.28$. The values obtained for the different frequencies and polarizations of the microwave link, which are listed in Table 2, are in good agreement with Berne and Uijlenhoet (2007).

To parameterize the errors $\Delta \ln (\boldsymbol{\alpha})$, assumed uncorrelated, we can proceed in the following way. First, we simulate the specific attenuation using the rain rate estimated by the radar (on the closest pixel) with the values of $\alpha$ and $\beta$ of Table 2. Hence, denoting $k_{l}$ the specific attenuation measured by the link and $k_{r}$ the specific attenuation simulated by the radar, we compute the error of the specific attenuation:

$$
\Delta \ln (k)=\sigma\left[\ln \left(k_{l}\right)-\ln \left(k_{r}\right)\right] .
$$

Thus, based on Eq. (23) we can define

$$
\Delta \ln (\alpha)=\sqrt{\Delta \ln (k)^{2}-\beta^{2} \Delta \ln \left(r_{r}\right)^{2}} .
$$

In this way, we obtain $\Delta \alpha \approx \Delta \ln (\alpha) \times \alpha$ around $0.13,0.36$, and 0.60 for the link at 23,38 , and $58 \mathrm{GHz}$ respectively.

\section{c. Rain rate retrieval}

Figure 4 shows the rain rate estimated by the radar (i.e., the prior) and the rain rate retrieved using the
TABLE 2. The $\alpha$ and $\beta$ values from DSD data and scattering simulation.

\begin{tabular}{ccccc}
\hline \hline Frequency & $\alpha_{H}$ & $\beta_{H}$ & $\alpha_{V}$ & $\beta_{V}$ \\
\hline $23 \mathrm{GHz}$ & 0.12 & 1.06 & 0.10 & 1.01 \\
$38 \mathrm{GHz}$ & 0.29 & 0.93 & 0.25 & 0.91 \\
$58 \mathrm{GHz}$ & 0.45 & 0.81 & 0.40 & 0.80 \\
\hline
\end{tabular}

proposed variational approach for a given time step during three different events: 19 June 2009 at 1805 UTC, 17 July at 2205 UTC, and 10 October at 1205 UTC. For the first case, the assimilation of rain gauge and link data globally increases the rain rate values: see, for instance, the pixel $(11,11)$, in which a rain gauge is located, and the pixel $(8,19)$, covered by a link. But this increase is not uniform, and the rain rate in the central zone even decreases after assimilation. For the second case, the assimilation (in particular of the link information) results in a more contrasted spatial distribution of the rain rate values in the central zone. For the third case, the assimilation tends to globally lower the rain rate. Please note that the number of links available for assimilation is varying during the considered period, so the number of "link" pixels is varying from one case to the other.

Figure 5 shows the prior (from the simulation) and the retrieved values of $\alpha$ for the different links. It must be noted that the retrieved $\alpha$ values are constant along the path of each link. This can be interpreted as the fact that the variability due to the DSD is overwhelmed by other effects such as wet-antenna attenuation or transmission/ reception errors that are constant along the path. Moreover, the retrieved $\alpha$ values are similar to the prior values for links at $23 \mathrm{GHz}$, while they are systematically larger at 38 and even more at $58 \mathrm{GHz}$. The difference between prior and posterior alpha also depends on link length since link length and link frequency are highly negatively correlated. This behavior is observed for many time steps. This systematic bias in the retrieved values is likely related to systematic bias in the processing of the link data. For instance, using larger values of wetantenna attenuation decreases this bias. Further investigation of this behavior is, however, beyond the scope of this paper, and we decided to use the same wet-antenna attenuation value at all frequencies.

To examine the behavior of our problem, the cost function and the norm of the gradients are shown in Fig. 6. The cost function does not present any irregularity, and the system is only weakly nonlinear, so the Gauss-Newton scheme is stable. The norm of the gradients does not indicate any problem in the minimization. The Hessian was always invertible, so no extra steps were required.

The inverse of the Hessian matrix is the error covariance matrix of the solution, and the square root of 

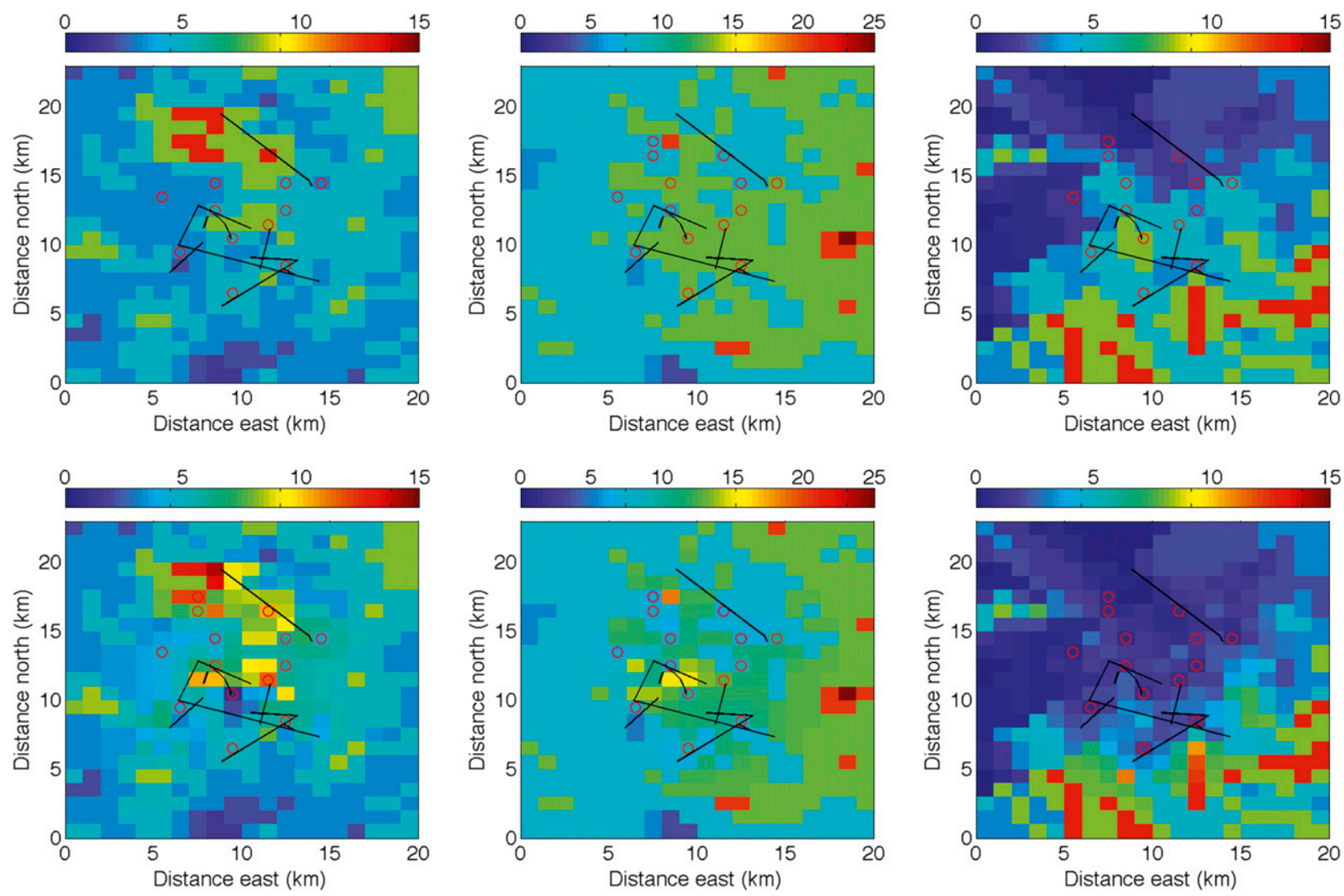

FIG. 4. (top) Rain rate $\left(\mathrm{mm} \mathrm{h}^{-1}\right)$ from the MeteoSwiss radar product at (left to right) 1805 UTC 19 Jun 2009,2205 UTC 17 Jul, and 1205 UTC 10 Oct. The red circles and the crosses indicate the rain gauge and microwave link locations used in the assimilation. (bottom) retrieved rain rate values $\left(\mathrm{mm} \mathrm{h}^{-1}\right)$ obtained by assimilating microwave link and rain gauge information recorded on the corresponding time steps. The radar is located at $(7,0)$.

the diagonal matrix gives the root-mean-square error (RMSE) in each individual retrieved variable (e.g., Rogers 2008). Figure 7 shows the spatial distribution of this error associated with the retrieved rain rate. Although theoretical, this error is useful to illustrate the decrease of uncertainty nearby the rain gauge and microwave link locations.

\section{d. Evaluation}

A cross-validation approach is used to quantify the performance of the proposed technique. The basic idea of the cross validation is to use only part of the available data during the assimilation, such that some of the data can be used as reference. To quantify the improvement in the rain rate estimates using the proposed method, a location where two rain gauges are close enough to be significantly correlated is used (otherwise, the assimilation does not modify the prior). One rain gauge is kept out of the assimilation and is later used to quantify the error in the prior and in the retrieved rain rate. This error is supposed to be larger than the error due to the comparison of a point and a pixel value. Table 3 shows the mean rain rate $\left(\mathrm{mm} \mathrm{h}^{-1}\right)$ of the five selected rain gauges for the three considered events.

Figure 8 shows the error in rain rate when rain gauge 3 (RG3) is kept out of the assimilation and is used as a reference for the prior (radar only) and the retrieved values (rain gauge and microwave link data assimilated). The mean error slightly increases, but remains below $0.1 \mathrm{~mm} \mathrm{~h}^{-1}$, while the standard deviation significantly decreases from 1.44 to $1.11 \mathrm{~mm} \mathrm{~h}^{-1}$, illustrating the improvement due to the assimilation.

To quantify the improvement in rain rate estimation, we can compute the efficiency (Nash and Sutcliffe 1970) defined as

$$
E=1-\frac{\sum_{t=1}^{n}\left(r_{t}-r_{g_{t}}\right)^{2}}{\sum_{t=1}^{n}\left(\overline{r_{g_{t}}}-r_{g_{t}}\right)^{2}},
$$

where $r_{t}$ represents the retrieved rain rate, $r_{g_{t}}$ is the rain rate from the rain gauge, and $n$ is the total number of time steps. The efficiency values obtained during the 


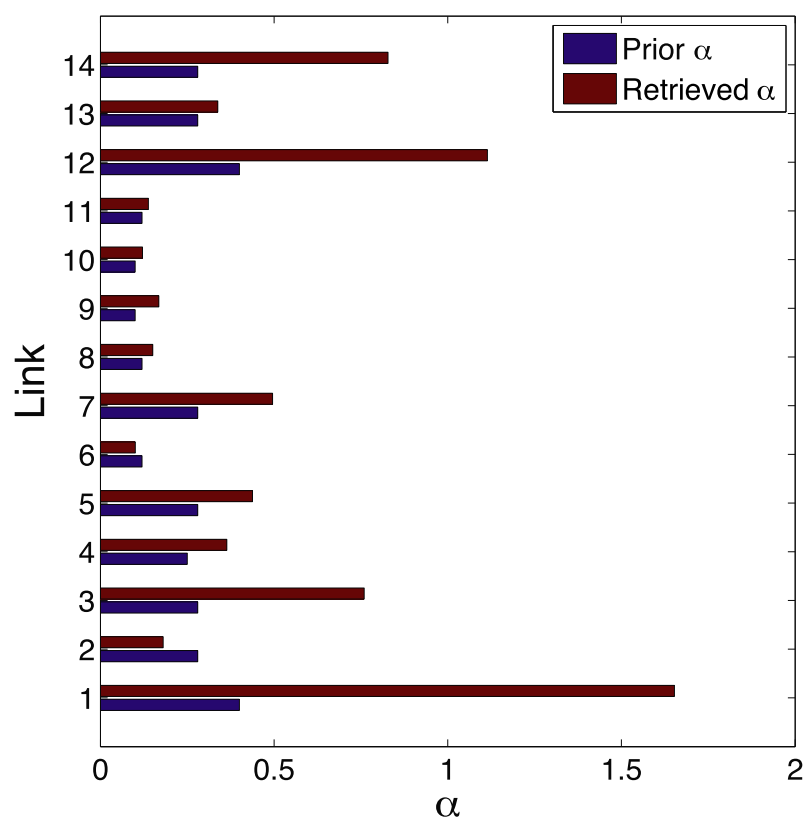

FIG. 5. Prior (blue) and retrieved (red) prefactor $\alpha$ of Eq. (2) at 1805 UTC 19 Jun 2009.

three considered events for the retrieved and prior rain rate values are listed in Table 4.

We also compute the normalized RMSE, defined as

$$
\text { RMSE }=\frac{\sqrt{\overline{\left(r_{t}-r_{g_{t}}\right)^{2}}}}{\overline{r_{g_{t}}}} .
$$

The RMSE values obtained during the three considered events for the retrieved and prior (indirectly) rain rate values are listed in Table 5.

From Tables 4 and 5, the added value of the assimilation is evident. The cross validation at the rain gauges RG3 and RG4, which are located in an area with no microwave link nearby, shows an improvement in the rain rate after the assimilation. The efficiency increases from $15 \%$ to $42 \%$ and the RMSE decreases from $20 \%$ to $30 \%$. An improvement in both $E$ and RMSE after assimilation (although more limited) is also seen at the locations of RG9 and RG12, where there are both rain gauges and microwave links. Finally, the cross validation at the location of RG11, where there is no rain gauge nearby, shows that the assimilation of link data is also beneficial for the retrieved rain rate values: efficiency increases from $10 \%$ to $30 \%$ and RMSE decreases from 1 to $23 \%$.

The efficiency values show a global improvement in the retrieved rain rate: both criteria are better in 15 cases out of 15 , with an increase in efficiency of about of $12 \%$


FIG. 6. (top) The cost function and (bottom) the norm of the gradients are shown for the first 60 iterations, but fewer iterations are necessary to reach a good approximation of the minimum since after 10 iterations the changes in the cost function are smaller than $0.1 \%$.

on average and up to $42 \%$; RMSE values show decreases of about $-16 \%$ and down to $-31 \%$. It must be noted that the prior is good, likely because of the fact that the Albis' radar is close by. The quality of the prior could be lower further from the radar, when the vertical variability is large (e.g., localized bright band), and the improvement in rain rate by combining radar, rain gauge, and microwave link measurements is expected to be even larger.

\section{Conclusions}

A variational method has been used to retrieve rain rate by combining rain gauge, microwave link, and weather radar data and to retrieve the coefficient $\alpha$ of the $k-r$ relationship simultaneously, as well as the 


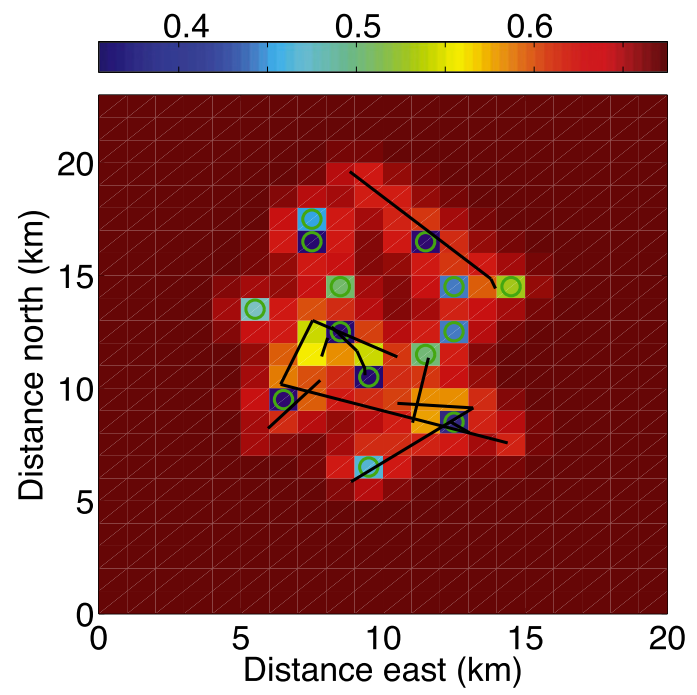

FIG. 7. Standard deviation of the error in the retrieved rain rate, in logarithm scale. The prior error of the rain rate (in log) was 0.68 everywhere. Notice how the error decreases to 0.4 where both gauge and microwave are located; this means an error of 0.4 times rain rate retrieved $\left(\mathrm{mm} \mathrm{h}^{-1}\right)$.

associated uncertainty. All the covariance matrices have been carefully parameterized using data from rain gauges and weather radars collected during several months. In addition, measurements from a disdrometer have been used to simulate the specific attenuation values in order to estimate the specific attenuation error and to initialize the parameters of the $k-r$ relationship.

The main limitations of this approach lie in the necessity to have nonzero positive values as prior since we retrieve logarithmic values and in the parameterization of the covariances matrices, which is needed if the algorithm is applied in another climatic region. It might also be difficult to obtain microwave link data from telecommunication companies.

This methodology is computationally fast and shows an improvement of $12 \%$ on average and up to $42 \%$ in efficiency and a decrement of $-16 \%$ on average and down to $-31 \%$ of the RMSE of the retrieved rain rate in a region in which the radar already provides good prior

TABLE 3. Mean rain rate $\left(\mathrm{mm} \mathrm{h}^{-1}\right)$ for the three events that are used for the cross validation.

\begin{tabular}{lccc}
\hline \hline Events & 19 June & 17-18 July & 10 October \\
\hline RG3 & 1.15 & 1.84 & 0.45 \\
RG4 & 1.26 & 1.95 & 0.46 \\
RG9 & 5.75 & 3.79 & 1.05 \\
RG12 & 2.99 & 1.74 & 0.51 \\
RG11 & 2.29 & 1.69 & 0.72 \\
\hline
\end{tabular}
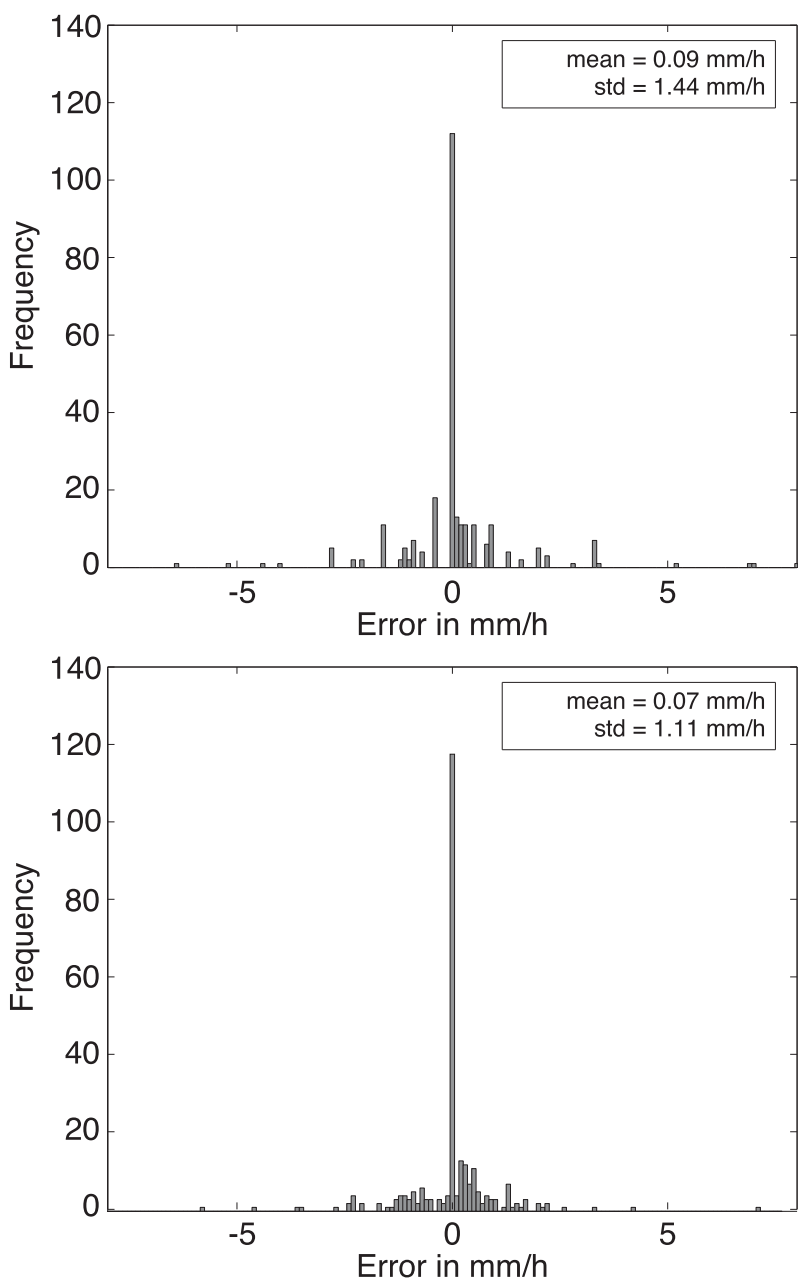

FIG. 8. Distribution of the error for RG3 (top) before and (bottom) after the assimilation.

information. Thus, we expect that the assimilation of different sources of information in our statistical framework can significantly improve the rain rate estimates in regions where the prior information provided by radar is poor. Future work might focus on investigating the microwave error correlations to enhance the characterization of the microwave link errors.

TABLE 4. Efficiency values after the assimilation and the relative deviation from the prior for the three considered events.

\begin{tabular}{llll}
\hline \hline Events & \multicolumn{1}{c}{ 19 June } & \multicolumn{1}{c}{$17-18$ July } & \multicolumn{1}{c}{ 10 October } \\
\hline RG3 & $0.70(+20.8 \%)$ & $0.63(+37.7 \%)$ & $0.78(+24 \%)$ \\
RG4 & $0.72(+16.3 \%)$ & $0.71(+15.4 \%)$ & $0.43(+41.6 \%)$ \\
RG9 & $0.60(+6.5 \%)$ & $0.63(+9.8 \%)$ & $0.68(+3.5 \%)$ \\
RG12 & $0.80(+2.8 \%)$ & $0.53(+3.4 \%)$ & $0.37(+9.6 \%)$ \\
RG11 & $0.49(+13.3 \%)$ & $0.78(+10 \%)$ & $0.56(+30.5 \%)$ \\
\hline
\end{tabular}


TABLE 5. RMSE values after the assimilation and the relative deviation from the prior for the three considered events.

\begin{tabular}{llll}
\hline \hline Events & \multicolumn{1}{c}{ 19 June } & \multicolumn{1}{c}{$17-18$ July } & 10 October \\
\hline RG3 & $0.96(-23.5 \%)$ & $0.76(-29.8 \%)$ & $2.48(-30.8 \%)$ \\
RG4 & $0.87(-20.5 \%)$ & $0.63(-19.1 \%)$ & $2.93(-23.8 \%)$ \\
RG9 & $1.26(-7.6 \%)$ & $1.13(-11.1 \%)$ & $1.44(-5.1 \%)$ \\
RG12 & $1.47(-6.3 \%)$ & $1.36(-3.4 \%)$ & $2.71(-6.8 \%)$ \\
RG11 & $1.54(-11 \%)$ & $0.97(-17.1 \%)$ & $2.16(-23.3 \%)$ \\
\hline
\end{tabular}

Acknowledgments. We thank Marc Schneebeli for valuable discussions and for providing the codes to run the scattering simulations. The radar rain product, the RSL, and the rain gauge data were kindly provided by Jörg Rieckermann from EAWAG. The financial support from the Swiss National Science Foundation for the COMCORDE project is acknowledged (Grant CR22D135551).

\section{REFERENCES}

Andsager, K., K. V. Beard, and N. F. Laird, 1999: Laboratory measurements of axis ratios for large rain drops. J. Atmos. Sci., 56, 2673-2683.

Atlas, D., and C. W. Ulbrich, 1977: Path and area integrated rainfall measurement by microwave attenuation in the $1-3 \mathrm{~cm}$ band. J. Appl. Meteor., 16, 327-332.

Barber, P., and C. Yeh, 1975: Scattering of electromagnetic waves by arbitrarily shaped dielectric bodies. Appl. Opt., 14, 2864-2872.

Battan, L. J., 1973: Radar Observation of the Atmosphere. University of Chicago Press, 324 pp.

Beard, K. V., 1977: Terminal velocity adjustment for cloud and precipitation drops aloft. J. Atmos. Sci., 34, 1293-1298.

Berenguer, M., and I. Zawadzki, 2008: A study of the error covariance matrix of radar rainfall estimates in stratiform rain. Wea. Forecasting, 23, 1085-1101.

Berne, A., and R. Uijlenhoet, 2007: Path-averaged rainfall estimation using microwave links: Uncertainty due to spatial rainfall variability. Geophys. Res. Lett., 34, L07403, doi:10.1029/2007GL029409.

Bianchi, B., P.-J. van Leeuwen, R. J. Hogan, and A. Berne, 2013: Quality control of rain gauge measurements using telecommunication microwave links. J. Hydrol., 492, 15-23, doi:10.1016/ j.jhydrol.2013.03.042.

Brandes, E., G. Zhang, and J. Vivekanandan, 2004: Drop size distribution retrieval with polarimetric radar: Model and application. J. Appl. Meteor., 43, 461-475.

Bringi, V. N., and V. Chandrasekar, 2001: Polarimetric Doppler Weather Radar. Cambridge University Press, 662 pp.

Caumont, O., V. Ducrocq, É. Wattrelot, G. Jaubert, and S. PradierVabre, 2010: 1D+3DVar assimilation of radar reflectivity data: A proof of concept. Tellus, 62A, 173-187.

Chahine, M. T., and Coauthors, 2006: AIRS: Improving weather forecasting and providing new data on greenhouse gases. Bull. Amer. Meteor. Soc., 87, 911-926.

D'Amico, M., M. Pinotti, and C. Capsoni, 2003: The MANTISSA project: First results from the Italian field experiments. IGARSS '03: Proceedings of the 2003 IEEE International
Geoscience and Remote Sensing Symposium, Vol. 7, IEEE, 4314-4316, doi:10.1109/IGARSS.2003.1295500.

Fletcher, S. J., and M. Zupanski, 2006: A data assimilation method for log-normally distributed observational errors. Quart. J. Roy. Meteor. Soc., 132, 2505-2519, doi:10.1256/ qj.05.222.

Germann, U., G. Galli, M. Boscacci, and M. Bolliger, 2006: Radar precipitation measurement in a mountainous region. Quart. J. Roy. Meteor. Soc., 132, 1669-1692, doi:10.1256/qj.05.190.

Goldshtein, O., H. Messer, and A. Zinevich, 2009: Rain rate estimation using measurements from commercial telecommunication links. IEEE Trans. Signal Process., 57, 1616-1625.

Goudenhoofdt, E., and L. Delobbe, 2008: Evaluation of radargauge merging methods for quantitative precipitation estimates. Hydrol. Earth Syst. Sci., 13, 195-203.

Grum, M., S. Krämer, H. R. Verworn, and A. Redder, 2005: Combined use of point rain gauges, radar, microwave link and level measurements in urban hydrological modelling. Atmos. Res., 77, 313-321.

Hogan, R., 2007: A variational scheme for retrieving rainfall rate and hail reflectivity fraction from polarization radar. J. Appl. Meteor. Climatol., 46, 1554-1564.

Jaffrain, J., A. Studzinski, and A. Berne, 2011: A network of disdrometers to quantify the small-scale variability of the raindrop size distribution. Water Resour. Res., 47, W00H06, doi:10.1029/2010WR009872.

Krämer, S., H.-R. Verwon, and A. Redder, 2005: Improvement of X-band radar rainfall estimates using a microwave link. Atmos. Res., 77, 278-299.

Leijnse, H., R. Uijlenhoet, and J. N. M. Stricker, 2007: Rainfall measurement using radio links from cellular communication networks. Water Resour. Res., 43, W03201, doi:10.1029/ 2006WR005631.

McLaughlin, D., 2002: An integrated approach to hydrologic data assimilation: Interpolation, smoothing, and filtering. Adv. Water Resour., 25, 1275-1286, doi:10.1016/S0309-1708(02)00055-6.

Meischner, P. F., V. N. Bringi, D. Heimann, and H. Holler, 1991: A squall line in southern Germany: Kinematics and precipitation formation as deduced by advanced polarimetric and Doppler radar measurements. Mon. Wea. Rev., 119, 678-701.

Meissner, T., and F. J. Wentz, 2004: The complex dielectric constant of pure and sea water from microwave satellite observations. IEEE Trans. Geosci. Remote Sens., 42, 1836-1849.

Messer, H., A. Zinevich, and P. Alpert, 2006: Environmental monitoring by wireless communication networks. Science, 312, 713, doi: $10.1126 /$ science. 1120034 .

Mishchenko, M. I., L. D. Travis, and D. W. Mackowski, 1996: T-matrix computations of light scattering by nonspherical particles: A review. J. Quant. Spectrosc. Radiat. Transfer, 55, $535-575$.

Nash, J. E., and J. V. Sutcliffe, 1970: River flow forecasting through conceptual models part I-A discussion of principles. J. Hydrol., 10, 282-290.

Nespor, V., and B. Sevruk, 1999: Estimation of wind-induced error of rainfall gauge measurements using a numerical simulation. J. Atmos. Oceanic Technol., 16, 450-464.

Olsen, R. L., D. V. Rogers, and D. B. Hodge, 1978: The $\mathrm{aR}^{\mathrm{b}}$ relation in the calculation of rain attenuation. IEEE Trans. Antennas Propag., 26, 318-329.

Overeem, A., H. Leijnse, and R. Uijlehoet, 2011: Measuring urban rainfall using microwave links from commercial cellular communication networks. Water Resour. Res., 47, W12505, doi:10.1029/2010WR010350. 
Rahimi, A. R., G. J. G. Upton, A. R. Holt, and R. J. Cummings, 2003: Use of dual-frequency microwave links for measuring path-averaged rainfall. J. Geophys. Res., 108, 4467, doi:10.1029/ 2002JD003202.

,$- \ldots$, and — 2004: Dual-frequency links_A complement to gauges and radar for the measurement of rain. J. Hydrol., 288, 3-12.

Rogers, C. D., 2008: Inverse Methods for Atmospheric Sounding. 3rd ed. World Scientific, $240 \mathrm{pp}$.

Ryzhkov, A. V., T. J. Schuur, D. W. Burgess, P. L. Heinselman, S. E. Giangrande, and D. S. Zrnic, 2005: The joint polarization experiment, polarimetric rainfall measurements, and hydrometeor classification. Bull. Amer. Meteor. Soc., 86, 809-824.

Sauvageot, H., 1994: The probability density function of rain rate and the estimation of rainfall by area integrals. J. Appl. Meteor., 33, 1255-1262.

Schneebeli, M., and A. Berne, 2012: An extended Kalman filter framework for polarimetric X-band weather radar data processing. J. Atmos. Oceanic Technol., 29, 711-730.
Sieck, L. C., S. J. Burges, and M. Steiner, 2007: Challenges in obtaining reliable measurements of point rainfall. Water Resour. Res., 43, W01420, doi:10.1029/2005WR004519.

Thurai, M., and V. N. Bringi, 2005: Drop axis ratios from a 2D video disdrometer. J. Atmos. Oceanic Technol., 22, 966-978.

Upton, G. J. G., and A. Rahimi, 2003: On-line detection of errors in tipping-bucket rain gauges. J. Hydrol., 278, 197-212.

World Meteorological Organization, 2008: Guide to Meteorological Instruments and Methods of Observation. 7th ed. WMO Series, No. 8, World Meteorological Organization, $681 \mathrm{pp}$

Zinevich, A., P. Alpert, and H. Messer, 2008: Estimation of rainfall fields using commercial microwave communication networks of variable density. Adv. Water Resour., 31, 1470-1480, doi:10.1016/j.advwatres.2008.03.003.

,-- , and — 2009: Frontal rainfall observation by commercial microwave communication network. J. Appl. Meteor. Climatol., 48, 1317-1334. 


\title{
CORRIGENDUM
}

\author{
BLANDINE BIANCHI \\ Environmental Remote Sensing Laboratory, Civil and Environmental Engineering, School of Architecture, \\ Ecole Polytechnique Federale de Lausanne, Lausanne, Switzerland \\ Peter JAN VAn LeEuwen AND Robin J. Hogan \\ Department of Meteorology, University of Reading, Reading, United Kingdom
}

AleXis BERNE

Environmental Remote Sensing Laboratory, Civil and Environmental Engineering, School of Architecture, Ecole Polytechnique Federale de Lausanne, Lausanne, Switzerland

There is an error in the References section of Bianchi et al. (2013a), which appeared in the December 2013 issue of the Journal of Hydrometeorology. The reference "Bianchi et al. (2013)" appearing on p. 1908 had incorrect coauthors listed. The correct reference appears as Bianchi et al. (2013b) below.

The staff of the Journal of Hydrometeorology regrets any inconvenience this error may have caused.

\section{REFERENCES}

Bianchi, B., P.-J. van Leeuwen, R. J. Hogan, and A. Berne, 2013a: A variational approach to retrieve rain rate by combining information from rain gauges, radars, and microwave links. J. Hydrometeor., 14, 1897-1909, doi:10.1175/JHM-D-12-094.1.

_ J. Rieckermann, and A. Berne, 2013b: Quality control of rain gauge measurements using telecommunication microwave links. J. Hydrol., 492, 15-23, doi:10.1016/j.jhydrol.2013.03.042.

Corresponding author address: Alexis Berne, Environmental Remote Sensing Laboratory, Civil and Environmental Engineering, School of Architecture, Ecole Polytechnique Fédérale de Lausanne, GR C2 564, Station 2, CH-1015 Lausanne, Switzerland.

E-mail: alexis.berne@epfl.ch 